\title{
Brain Glioblastoma
}

National Cancer Institute

\section{Source}

National Cancer Institute. Brain Glioblastoma. NCI Thesaurus. Code C4642.

A WHO grade IV malignant astrocytic tumor that arises from the brain, usually the cerebral hemispheres. It is characterized by the presence of poorly differentiated astrocytes, cellular polymorphism, nuclear atypia, and increased mitotic activity. The prognosis is poor. 